CLINICAL STUDY

\title{
Distribution of adiponectin multimeric forms in Chinese women with polycystic ovary syndrome and their relation to insulin resistance
}

\author{
Tao Tao, Edmond P Wickham III ${ }^{1}$, WuQiang Fan, Jiejin Yang and Wei Liu \\ Department of Medicine and Endocrinology, Renji Hospital, Shanghai Jiaotong University School of Medicine, 1630 Dongfang Road, Pudong, Shanghai \\ 200127, People's Republic of China and ${ }^{1}$ Division of Endocrinology and Metabolism, Department of Internal Medicine, Virginia Commonwealth \\ University, Richmond, Virginia 23227, USA \\ (Correspondence should be addressed to W Liu; Email: sue_liuwei@163.com)
}

\begin{abstract}
Objective: Adiponectin, an abundant adipokine with insulin-sensitizing properties, exists in different multimeric forms, including low-molecular weight, medium-molecular weight, and high-molecular weight (HMW) species. Alterations in the distribution of adiponectin multimers and the relationship between adiponectin multimers and insulin resistance (IR) in women with polycystic ovary syndrome (PCOS) remain unclear. The objective of this study was to compare adiponectin multimerization status and estimate insulin sensitivity in Chinese women with PCOS compared with age- and body mass index (BMI)-matched controls.

Methods: Cross-sectional study involving 64 Chinese women with PCOS and 59 normal women. Circulating total adiponectin and its multimeric forms were determined by ELISA, and IR was estimated using the homeostasis model assessment IR index (HOMA-IR).

Results: After controlling for BMI status, levels of both total and HMW adiponectin were significantly lower in women with PCOS compared with normal women $(P<0.05)$. Furthermore, HMW adiponectin provided a stronger contribution to models predicting IR than total adiponectin. Lastly, decreased HMW adiponectin was associated with increased HOMA-IR in both normal and PCOS women, and this association was independent of both overall adiposity and visceral adiposity.

Conclusion: Levels of both total and HMW adiponectin were decreased in Chinese women with PCOS compared with normal control women, and the differences in HMW adiponectin persisted after controlling for BMI. Furthermore, HMW adiponectin is a stronger predictor of IR than total adiponectin in both women with PCOS and normal women.
\end{abstract}

European Journal of Endocrinology 163 399-406

\section{Introduction}

Polycystic ovary syndrome (PCOS) is one of the most common endocrine diseases in reproductive age women, and is a common cause of menstrual dysfunction, infertility, and hirsutism (1). Insulin resistance (IR) and obesity play a central role in the pathogenesis of PCOS $(2,3)$. In addition to the burden of IR attributed to obesity, women with PCOS demonstrate a form of IR intrinsic to the syndrome (4). However, the mechanism responsible for the IR intrinsic to the syndrome is unclear (5). Therefore, an important consideration is whether adipocytokines such as adiponectin, a potential mediator of insulin sensitivity (6), are also implicated in the pathogenesis of PCOS. Levels of adiponectin, an abundant adipocyte-derived cytokine, are strongly correlated with measures of insulin sensitivity $(7,8)$, and low levels of adiponectin have been associated with future risk of cardiovascular disease and type 2 diabetes $(9,10)$. In recent years, the relationship between the physiological action and structure of adiponectin has attracted wide attention.

Adiponectin circulates in different multimer complexes, specifically classified as high-molecular weight (HMW) multimers, medium-molecular weight hexamers (MMW), and low-molecular weight (LMW) trimers (11). Recent clinical studies have revealed that the HMW complex is the most biologically potent form, and plays a key role in the regulation of IR $(12,13)$. Although the relationship between alterations in adiponectin multimerization and IR in women with PCOS compared with normal women remains unclear, we hypothesized that the distribution of adiponectin multimeric forms, in particular the HMW form, is decreased in women with PCOS compared with normal control women. We further hypothesized that this difference is independent of obesity, and that alterations in HMW adiponectin contribute to the IR intrinsic to the syndrome. 
Thus, in the present study, we evaluated adiponectin multimerization status and estimates of insulin sensitivity in Chinese women with PCOS compared with ageand body mass index (BMI)-matched controls.

\section{Materials and methods}

Sixty-four women with PCOS (43 obese women and 21 normal weight women) from Shanghai, China and 59 normal cycling, non-hirsute women (38 obese women and 21 normal weight women) between 16 and 35 years of age were enrolled in the study. PCOS was defined using the 1990 National Institutes of Health criteria (14). Pregnancy was excluded by a urinary pregnancy test. All women had normal thyroid function and prolactin levels, and late-onset nonclassic congenital hyperplasia was excluded by a basal $17-\alpha$ hydroxyprogesterone value of $<300 \mathrm{ng} / \mathrm{dl}$ (15). Women receiving glucocorticoids, antiandrogens, or oral contraceptives within the previous 30 days, or ovulation induction agents, antiobesity medications, or insulin-sensitizing agents within the previous 60 days were excluded. All women were evaluated by transvaginal ultrasonography to define ovarian morphology (16). Control subjects were screened by medical history, physical examination, laboratory evaluation, and transvaginal ultrasound. Normal weight women were defined as those with a BMI $<25 \mathrm{~kg} / \mathrm{m}^{2}$, and obesity was defined as those with a BMI of $\geq 25 \mathrm{~kg} / \mathrm{m}^{2}$ according to the 2000 WHO-WPR criteria (17). Women with PCOS and control women were matched for age, BMI, and fat mass. All study evaluations and procedures were conducted in accordance with the guidelines of Helsinki Declaration on human experimentation. The study was approved by the ethics committee of Shanghai Renji Hospital, and all subjects provided written informed consent.

\section{Anthropometric measurements}

The height and weight of each subject wearing light clothing was measured to the nearest $0.1 \mathrm{~cm}$ and $0.1 \mathrm{~kg}$ respectively using a digital scale and stadiometer. BMI was calculated as body weight $(\mathrm{kg})$ divided by height $(\mathrm{m})$ squared. Waist circumference (WC) and hip circumference (HC) were measured by a single individual. WC was determined by measuring the circumference at the narrowest point between the lower border of the rib cage and the iliac crest. $\mathrm{HC}$ was determined by measuring the circumference at the level of the symphysis pubis and the greatest gluteal protuberance. The waist-to-hip ratio (WHR) was then calculated by dividing the WC by the HC. Percent body fat was assessed by foot-to-foot measures of bioelectrical impedance obtained using a TBF-300 body composition analyzer (TANITA, UK Ltd, Middlesex, UK, www.tanita.com).

\section{Laboratory assays}

All laboratory evaluations were performed at $0800 \mathrm{~h}$ after an overnight fast during the early follicular phase (days 2-5) of a spontaneous menstrual cycle, except in subjects with amenorrhea $>3$ months who were examined randomly. Fasting glucose and insulin samples were stored at $4{ }^{\circ} \mathrm{C}$, and analyzed on the day of analysis. All serum samples for total adiponectin and adiponectin multimeric forms were stored at $-70{ }^{\circ} \mathrm{C}$ until assayed.

Competitive electrochemiluminescence immunoassays on the Elecsys autoanalyzer 2010 (Roche Diagnostics) were used to quantify serum total testosterone. Sex hormone-binding globulin (SHBG) levels were measured by chemiluminescent immunoassay (Elecsys autoanalyzer 2010, Roche Diagnostics) validated for plasma SHBG (18). The coefficient of variation (CV) for SHBG using this methodology was $6 \%$. Free testosterone values were calculated based on total testosterone and SHBG levels according to the method outlined by Vermeulen et al. (19) assuming an albumin concentration of $4 \mathrm{~g} / \mathrm{dl}$ (http://www.issam.ch/freetesto.htm). Plasma glucose was determined using the glucose oxidase methodology. All measurements were performed with Roche reagents (D 2400 and E 170 Modular Analytics modules with Roche/Hitachi analyzers; Roche Diagnostics). Insulin levels were measured by RIA. The intra-assay CV of insulin and of steroid hormone assays were 5.5 and $<10 \%$ respectively. To estimate IR, the homeostasis model assessment IR index (HOMA-IR) was calculated according to the formula: fasting serum insulin $(\mu \mathrm{UI} / \mathrm{ml}) \times$ fasting plasma glucose $(\mathrm{mmol} / \mathrm{l}) / 22.5(20)$.

Serum total adiponectin, HMW adiponectin, and combined HMW + MMW adiponectin levels were assayed directly in the same plate using a doublemonoclonal sandwich ELISA method (Daiichi Pure Chemicals, Tokyo, Japan, distributed by ALPCO diagnostics). To detect HMW adiponectin, serum samples were pretreated with a protease that selectively digested MMW and LMW adiponectin species. The combined HMW and MMW adiponectin concentrations were determined by pretreating the samples with a protease that specifically digested LMW adiponectin. MMW adiponectin concentrations were obtained by subtracting the HMW adiponectin value from the combined HMW + MMW value. Finally, the LMW adiponectin value was computed by subtracting HMW and MMW adiponectin values from the total adiponectin values. This specific assay for adiponectin multimers demonstrates a sensitivity of $0.04 \mathrm{ng} / \mathrm{ml}$; an inter-assay CV $<15 \%$; and an intra-assay CV of 5.3, 3.3, and $4.1 \%$ for total, HMW-adiponectin, and MMW + HMW adiponectin respectively (21). 


\section{Statistical analysis}

Results that were not normally distributed, based on the normal quartile plot, were log-transformed for all statistical analyses and reported back-transformed in their original units. All results were reported as means, or geometric means for log-transformed variables, with $95 \%$ confidence intervals (CIs). $P$ values $<0.05$ were considered significant. All statistical analyses were performed using JMP 8.0 software (SAS Institute, Cary, NC, USA).

For continuous variables, subgroup means were compared with one-way ANOVA testing considering four subgroups of women: lean control (LC), obese control (OC), lean PCOS (LP), and obese PCOS (OP). Tukey's honestly significantly difference test was performed when the result from the ANOVA was statistically significant. The relationships between levels of total and HMW adiponectin and hormonal and metabolic variables were evaluated by Spearman's correlation tests. Two forward, stepwise, multiple linear regression analyses were performed. The first model was performed to determine the best model to predict IR as estimated by HOMA-IR considering biologically plausible predictors, i.e. PCOS status, age BMI, WHR, total adiponectin, HMW adiponectin, and LMW adiponectin. These independent variables were selected based on literature, and because their correlations with insulin sensitivity were significant in the univariate analyses. Based on the results of the first model, a second model was created to determine the best model to predict serum levels of HMW adiponectin considering PCOS status, age, BMI, WHR, and free testosterone as plausible independent variables.

\section{Results}

The clinical characteristics and biochemical variables for the four groups of women according to PCOS and BMI status are summarized in Table 1. As expected, mean BMI $(F(3,119)=59.50, P<0.0001)$ and percent body fat $(F(3,119)=83.61, P<0.0001)$ were significantly different between groups, with higher mean values in both groups of obese women compared with groups of lean women. However, there were no significant differences in BMI or percent body fat in LC compared with the LP group or in OC group compared with the OP group. Similarly, WC $(F(3,119)=57.77, P<0.0001)$, HC $(F \quad(3,119)=43.38, P<0.0001)$, and WHR $(F(3,119)=64.12, \quad P<0.0001)$ were significantly different between groups. Specifically, mean WC, $\mathrm{HC}$, and WHR values were greater in the obese groups (OC and OP) compared with the lean groups (LC and LP) of women. As with BMI, there were no significant differences in WC, HC, or WHR between control and PCOS women according to BMI status. Mean total and free testosterone values were also significantly different between groups $(F \quad(3,119)=4.81, \quad P=0.003$; $F(3,119)=10.45, \quad P<0.0001$ respectively). Total testosterone values were significantly higher in the LP group compared with the other three groups. Free testosterone values were highest in the OP and LP groups and lowest in the LC groups. Significant differences in fasting glucose levels were noted between groups $(F(3,119)=9.74, P<0.001)$ with higher mean glucose values in the OP group and lower glucose values in the LC group. Mean HOMA-IR values were significantly different between groups $(F(3,119)=61.72$,

Table 1 Clinical and biochemical variables in women with polycystic ovary syndrome (PCOS) and normal, control women according to BMI status. Data are means (95\% confidence interval) unless noted.

\begin{tabular}{|c|c|c|c|c|c|}
\hline Variable & Lean control & Obese control & Lean PCOS & Obese PCOS & $P$ value \\
\hline$n$ & 21 & 38 & 21 & 43 & NA \\
\hline Age (years) & $21.9(18.7-25.0)$ & $26.0(23.7-28.3)$ & $22.9(19.7-26.0)$ & $25.9(23.7-28.0)$ & 0.08 \\
\hline $\mathrm{BMI}\left(\mathrm{kg} / \mathrm{m}^{2}\right)$ & $20.5(18.8-22.2)^{a, c}$ & $30.9(29.6-32.2)^{b, d}$ & $20.0(18.3-21.7)^{a, c}$ & $29.8(28.6-31.0)^{b, d}$ & $<0.0001$ \\
\hline Percent body fat & $23.1(20.4-25.9)^{a, c}$ & $42.7(40.7-44.8)^{b, d}$ & $24.8(22.0-27.5)^{\mathrm{a}, \mathrm{c}}$ & $43.3(41.4-45.2)^{b, d}$ & $<0.0001$ \\
\hline Waist circumference $(\mathrm{cm})$ & $66.1(61.8-70.3)^{a, c}$ & $92.4(89.2-95.6)^{b, d}$ & $67.8(63.6-72.1)^{a, c}$ & $90.3(87.4-93.3)^{\mathrm{b}, \mathrm{d}}$ & $<0.0001$ \\
\hline Hip circumference (cm) & $86.7(83.3-90.1)^{\mathrm{a}, \mathrm{c}}$ & $105.8(103.3)^{\mathrm{b}, \mathrm{d}}$ & $86.9(83.5-90.3)^{a, c}$ & $102.3(99.9-104.7)^{b, d}$ & $<0.0001$ \\
\hline WHR & $0.76(0.74-0.79)^{\mathrm{a}, \mathrm{c}}$ & $0.87(0.85-0.89)^{b, d}$ & $0.78(0.76-0.80)^{a, c}$ & $0.88(0.87-0.90)^{b, d}$ & $<0.0001$ \\
\hline Total cholesterol (mmol/l) & $3.56(3.27-3.85)^{a, b, c}$ & $4.16(3.95-4.38)^{c, d}$ & $4.44(4.15-4.74)^{d}$ & $4.69(4.49-4.89)^{a, d}$ & $<0.0001$ \\
\hline HDL cholesterol (mmol/l) & $1.24(1.10-1.38)$ & $1.21(1.10-1.31)$ & $1.43(1.29-1.57)$ & $1.31(1.21-1.40)$ & 0.08 \\
\hline LDL cholesterol (mmol/l) & $2.29(2.21-2.38)^{a, c}$ & $2.84(2.63-3.04)^{d}$ & $2.68(2.38-2.97)$ & $2.97(2.70-3.24)^{d}$ & 0.003 \\
\hline Triglycerides $(\mathrm{mmol} / \mathrm{l})$ & $0.96(0.68-1.23)^{c}$ & $1.32(1.13-1.52)$ & $1.09(0.82-1.35)^{\mathrm{c}}$ & $1.65(1.47-1.84)^{b, d}$ & 0.0001 \\
\hline Fasting insulin* (mIU/l) & $9.98(8.37-11.90)^{\mathrm{a}, \mathrm{c}}$ & $20.14(17.67-22.95)^{\mathrm{b}, \mathrm{d}}$ & $10.01(8.39-11.93)^{a, c}$ & $18.84(16.67-21.31)^{\mathrm{b}, \mathrm{d}}$ & $<0.0001$ \\
\hline Fasting glucose $(\mathrm{mmol} / \mathrm{l})$ & $4.33(4.20-4.47)^{a, c}$ & $4.77(4.61-4.94)^{\mathrm{c}, \mathrm{d}}$ & $4.69(4.53-4.85)^{c}$ & $5.09(4.87-5.31)^{a, b, d}$ & $<0.0001$ \\
\hline HOMA-IR* & $1.92(1.59-2.32)^{\mathrm{a}, \mathrm{c}}$ & $4.25(3.69-4.89)^{b, d}$ & $2.03(1.38-2.45)^{a, c}$ & $4.22(3.70-4.83)^{b, d}$ & $<0.0001$ \\
\hline Total testosterone (nmol/l) & $1.97(1.49-2.46)^{b}$ & $2.30(1.94-2.66)^{b}$ & $3.21(2.73-3.70)^{\mathrm{a}, \mathrm{c}, \mathrm{d}}$ & $2.38(2.04-2.72)^{b}$ & 0.003 \\
\hline Free testosterone* $(\mathrm{pmol} / \mathrm{l})$ & $22.82(18.52-28.12)^{b, c}$ & $31.99(27.39-37.36)^{b}$ & $47.48(38.54-58.50)^{a, d}$ & $41.42(35.80-47.92)^{d}$ & $<0.0001$ \\
\hline
\end{tabular}

BMI, body mass index; HDL, high-density lipoprotein; HMW, high-molecular weight; HOMA-IR, homeostasis model assessment insulin resistance index; $P$ value for ANOVA for difference between groups. a $-<0.05$ compared with obese control, $\mathrm{b}-<0.05$ compared with lean $\mathrm{PCOS}, \mathrm{c}-<0.05$ compared with obese PCOS, and d $-<0.05$ compared with lean control. To convert cholesterol to $\mathrm{mg} / \mathrm{dl}$, divide by 0.02586 ; to convert triglycerides to mg/dl, divide by 0.01129 ; to covert glucose to $\mathrm{mg} / \mathrm{dl}$ :, divide by 0.05551 ; to convert total testosterone to $\mathrm{ng} / \mathrm{ml}$, divide by 3.467 ; to convert free testosterone to $\mathrm{pg} / \mathrm{ml}$, divide by 3.467 .

*Geometric means. 
$P<0.0001)$, with higher HOMA-IR values in women in the obese groups (OC and $\mathrm{OP}$ ) compared with the lean groups (LC and LP). However, significant differences in HOMA-IR values between OC and OP women or LC and LP women were not observed.
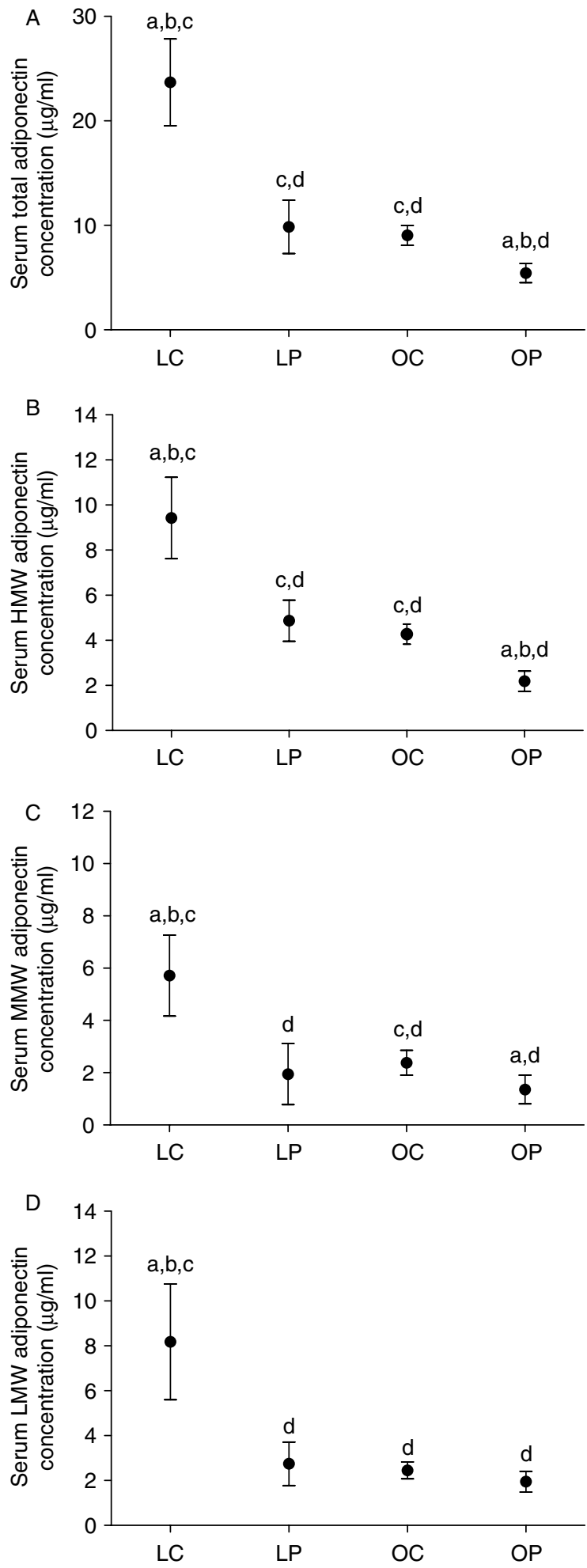

\section{Levels of total and three oligomeric forms of adiponectin}

Figure 1 illustrates the mean values of total adiponectin and the three multimeric forms of adiponectin for each group. Mean levels of total adiponectin were significantly different among groups $(F(3,119)=57.02$, $P<0.001)$ with the highest mean value observed in LC women $(22.11,95 \%$ CI (18.29-26.73)) and the lowest mean value observed in OP women (4.81 (4.22-5.50)). Total adiponectin levels were similar between women in the OC (8.59 (7.46-9.90)) and LP (8.56 (7.08-10.35)) groups. Mean HMW adiponectin values were also significantly different between groups $(F(3,119)=$ 43.51, $P<0.0001)$ with differences between groups similar to the pattern observed in total adiponectin (LC 8.52 (6.69-10.85), LP 4.58 (3.60-5.83), OC 4.05 (3.38-4.85), OP $1.72(1.45-2.03))$. Mean levels of MMW $(F(3,119)=17.32, P<0.0001)$ and LMW adiponectin $(F(3,119)=12.40, P<0.0001)$ also differed significantly between groups. Specifically, MMW and LMW adiponectin were highest in the LC women and lowest in the OP women.

When women with PCOS $(n=59)$ and normal control women $(n=64)$ were considered as whole groups, both levels of total $(F(1,121)=49.46, P<0.001)$ and HMW adiponectin $(F(1,121)=40.95, P<0.001)$ remained significantly decreased in women with PCOS compared with normal women. However, ratios of HMW to total adiponectin were not significantly different between women with PCOS and normal control women $(P=0.47)$.

\section{Correlation between adiponectin levels and clinical/biochemical characteristics}

Given the association between hypoadiponectinemia and increased adiposity, correlations between total and HMW adiponectin and anthropometric/metabolic parameters were preformed (Table 2). Considering all women $(n=123)$, both total and HMW adiponectin levels had inverse linear relationships with BMI, percent body fat, and WHR $(P<0.0001)$. Although neither total or HMW adiponectin levels correlated with total testosterone levels, both total and HMW adiponectin levels demonstrated inverse linear relationships with estimated free testosterone levels $(P<0.001$ and

Figure 1 Serum total adiponectin (A), HMW adiponectin (B), MMW adiponectin (C), and LMW adiponectin (D) concentrations in women with and without PCOS according to BMl category. Data are geometric means $(95 \% \mathrm{Cl})$. LC, lean control women $(n=21)$; LP, lean PCOS women ( $n=21)$; OC, obese control women $(n=38)$; OP, obese PCOS women $(n=43)$. HMW, high-molecular weight; LMW, low-molecular weight; MMW, middle molecular weight. $P$ value for ANOVA for difference between groups $<0.0001$ for total, HMW, MMW, and LMW adiponectin. a $-<0.05$ compared with obese control (OC), b-<0.05 compared with lean PCOS (LP), $c-<0.05$ compared with obese PCOS (OP), and d $-<0.05$ compared with lean control (LC). 
Table 2 Correlation between metabolic parameters and total and $\mathrm{HMW}$ adiponectin in normal control and polycystic ovary syndrome (PCOS) women.

\begin{tabular}{|c|c|c|c|c|}
\hline \multirow[b]{2}{*}{ Variable } & \multicolumn{2}{|c|}{ Total adiponectin ${ }^{a}$} & \multicolumn{2}{|c|}{ HMW adiponectin ${ }^{a}$} \\
\hline & Correlation & $P$ value & Correlation & $P$ value \\
\hline BM & 0.39 & $<0.0001$ & -0.35 & $<0.0001$ \\
\hline e & U. & & & \\
\hline WHR & -0.44 & $<0.0001$ & $-\mathrm{c}$ - $-2 x$ & 0.0001 \\
\hline erone & -0.09 & 0.35 & -0.06 & 0.51 \\
\hline SHBG & 0.60 & $<0.0001$ & 0.59 & $<0.0001$ \\
\hline Free te & 0.30 & $<0.001$ & -0.28 & 0.002 \\
\hline Total c & - & $<0.0001$ & -0.45 & $<0.0001$ \\
\hline HDL c & -0.07 & 0.44 & -0.09 & 0.31 \\
\hline LDL c & -0.3 & 0.0001 & -0 & $<0.001$ \\
\hline Triglycerides & -0.26 & 0.003 & -0.22 & 0.02 \\
\hline & -0.42 & $<0.0001$ & -0.39 & $<0.0001$ \\
\hline Fasting & -0.38 & $<0.0001$ & -0.37 & $<0.0001$ \\
\hline HOMA-I & -0.42 & $<0.0001$ & -0.40 & $<0.000$ \\
\hline
\end{tabular}

BMI, body mass index; HDL, high-density lipoprotein; HMW, high-molecular weight; HOMA-IR, homeostasis model assessment insulin resistance index LDL, low-density lipoprotein; SHBG, sex hormone-binding globulin; WHR, waist-to-hip ratio.

aLog-transformed prior to analysis.

$P<0.01$ respectively). Furthermore, strong positive linear correlation was observed between SHBG levels and both total and HMW adiponectin $(P<0.0001$ for both). Significant inverse linear relationships were also demonstrated between both total and HMW adiponectin and total cholesterol $(P<0.0001$ for both), low-density lipoprotein (LDL) cholesterol $(P<0.001$ for both), and triglycerides $(P=0.003$ and $P=0.02$ respectively). However, neither total nor HMW adiponectin levels correlated significantly with levels of high-density lipoprotein (HDL) cholesterol. Finally, both total and HMW adiponectin levels were found to have strong inverse linear relationships with fasting glucose, fasting insulin, and HOMA-IR values $(P<0.0001$ for all).

\section{Determinants of IR}

To investigate the independent determinants of IR among all women studied, we performed a forward, stepwise, multiple regression analysis with HOMA-IR as the dependent variable and PCOS status, age, BMI, WHR, total adiponectin, HMW adiponectin, and LMW adiponectin as potential contributors to IR. In the analysis, HMW adiponectin, BMI, and WHR, but not PCOS status or total adiponectin, were selected as independent variables for the final model of HOMA-IR. In the final model for HOMA-IR, the adjusted $R^{2}$ was $0.48(P<0.0001)$, and the regression coefficients $(\beta)$ were $-0.12(P=0.02)$ for HMW adiponectin, 0.04 $(P<0.0001)$ for BMI, and $1.64(P=0.02)$ for WHR. Thus, HMW adiponectin, BMI, and WHR were all independent predictors of IR as estimated by HOMA-IR. However, PCOS status and levels of total adiponectin did not appear to contribute significantly to the model for
HOMA-IR after controlling for the other potential independent variables.

Since percent body fat estimated by bioimpedence was strongly correlated with BMI $(r=0.93, P<0.001)$, both variables were not included in the model for IR to avoid multicollinearity. However, similar results were obtained if percent body fat was included as a dependent variable in the model in place of BMI (data not shown).

\section{Determinants of HMW adiponectin}

After HMW adiponectin was selected as a better predictor of HOMA-IR than total adiponectin in the first linear regression analysis, a second model was created, again using forward, stepwise, linear regression analysis, with HMW adiponectin as the dependent variable and age, PCOS status, free testosterone, BMI, and WHR as potential independent variables. Considering these variables, only free testosterone was not selected as an independent variable for the final model predicting HMW adiponectin. The adjusted $R^{2}$ for the model predicting HMW adiponectin was 0.40 $(P<0.0001)$, and age $(\beta=0.016, P=0.05)$, PCOS status $(\beta=-0.40, P<0.0001)$, and BMI $(\beta=-0.04$, $P<0.01)$, where all independent contributors to levels of HMW adiponectin in the final model.

\section{Discussion}

In this study, we investigated the distribution of circulating adiponectin oligomers in PCOS women compared with normal control women, and determined which multimeric forms of adiponectin correlated with IR. There are three principal findings in our study: i) after controlling for BMI status, levels of both total and HMW adiponectin levels were significantly lower in women with PCOS compared with those in normal women; ii) HMW adiponectin provided a stronger contribution to models predicting IR than total adiponectin among all women; and iii) decreased HMW adiponectin was associated with increased IR in both normal and PCOS women, and this association is independent of PCOS status, overall adiposity (BMI), and visceral adiposity (WHR).

Previous studies that assessed only total adiponectin in women with PCOS have yielded conflicting results $(22,23)$. However, it is noteworthy that adipocytes secret adiponectin as multimeric complexes, and different adiponectin isoforms appear to have unique regulatory effects on metabolic and inflammatory pathways $(24,25)$. In particular, the HMW form appears to have the most potent insulin-sensitizing effects. Recent clinical studies suggest that the HMW form of adiponectin is the primary mediator of the effects of adiponectin on insulin-stimulated glucose metabolism $(12,13,26,27)$. In these studies, only HMW adiponectin showed a similar close association 
with progression to type 2 diabetes, the degree of IR, and the presence of the metabolic syndrome. Therefore, we suggest that some of the inconsistencies in prior studies investigating alterations in adiponectin in PCOS women may be explained by the failure to assess levels of HMW adiponectin.

In this study, we demonstrated that women with PCOS have decreased levels of both total and HWM adiponectin, and that these alterations are independent of BMI. Furthermore, levels of HMW adiponectin demonstrated a strong inverse linear relationship with estimated levels of free testosterone. In the light of previous evidence demonstrating that testosterone inhibits the secretion of HMW adiponectin from rat adipocytes (28), elevated serum androgen levels may represent a plausible explanation for the observed differences in HMW adiponectin between PCOS and normal women. However, given the cross-sectional design of our study, causality cannot be established. Additionally, in a forward stepwise, linear regression analysis performed to determine predictors of HMW adiponectin in PCOS and normal women, free testosterone was no longer an independent predictor of HMW adiponectin after controlling for PCOS status, BMI, and age.

Furthermore, our results suggested that HMW adiponectin may be more important than total adiponectin in predicting IR in PCOS women and control women. The observed association between HMW adiponectin and IR is consistent with other studies suggesting that the HMW form of adiponectin has the most potent insulin-sensitizing properties $(12,13)$. At the onset of the study, we hypothesized that alterations in adiponectin multimers in women with PCOS may contribute to the IR intrinsic to the syndrome. Although we demonstrated that levels of HMW adiponectin were decreased in both lean and obese women with PCOS compared with BMI-matched controls, and that HMW adiponectin was a stronger predictor of IR than total adiponectin considering the entire study cohort, differences in IR as estimated by HOMA-IR were not observed between women with PCOS and control women in the unadjusted analysis. In the light of the well-described differences in IR previously observed between PCOS and normal women after controlling for BMI $(2,5)$, we suggest that using HOMA-IR as an estimate for IR, as opposed to other more robust methods, may have been unable to detect subtle differences in IR between groups and represents a potential limitation of the study.

Even though we did not observe significant differences in HOMA-IR between PCOS and normal women, SHBG levels were significantly lower in women with PCOS compared with normal women in this study. Although not a direct measure of IR, SHBG levels have been previously shown to correlate with insulin sensitivity measured by euglycemic clamp (29). Additionally, in this study, strong positive linear correlations were observed between both total and
HMW adiponectin and SHBG levels. Alternatively, the observation that total and HMW adiponectin were lower in women with PCOS compared with BMI-matched normal women, despite similar estimates of IR may suggest that alterations in adiponectin multimer distribution in women with PCOS contribute to the phenotypic presentation of the syndrome via additional mechanisms independent of influences on insulin sensitivity.

While there is ample literature regarding total adiponectin concentrations in patients with PCOS, data concerning the relative distribution of the different multimeric complexes of adiponectin in women with PCOS are limited (30). Furthermore, the studies reporting the relative distribution of adiponectin isoforms in patients with PCOS (31-33) have reported conflicting results. Aroda's (31) group previously reported that women with PCOS had lower total adiponectin levels compared with control women, and that the HMW species accounted for a smaller proportion of circulating adiponectin in women with PCOS. However, they did not examine the relationship between adiponectin multimer status and IR. Although Glintborg's group (32) also demonstrated lower total adiponectin levels in obese women with PCOS compared with age and BMI-matched control women, they failed to show significant differences in either HMW adiponectin levels or the ratio of HMW adiponectin to total adiponectin between the two groups of women. However, Glintborg et al. (32) did report a significant negative correlations between the HMW form of adiponectin and both WHR and insulin sensitivity. They also described changes in total and HMW adiponectin among PCOS patient randomized to receive pioglitazone or placebo for 14 weeks (32). Both total and HMW adiponectin levels increased with pioglitazone treatment, and the magnitude of change correlated with changes of insulin sensitivity. Barber et al. (33) compared adiponectin multimers between PCOS women and female controls, including a subset of 22 BMI- and fat mass-matched pairs, and discovered that both total and HMW adiponectin levels are lower in women diagnosed with PCOS. However, the differences in total and HMW adiponectin were no longer significant after adjusting for age and fat mass. As a result, they concluded that the differences in adiponectin multimers between the groups of women were explained by differences in fat mass and not by PCOS status. Consequently, they suggest that adiponectin does not play a central role in the pathophysiology of PCOS independent of adiposity.

The findings of the present study contrast with the results of two of these reports $(32,33)$. Several explanations for the alleged disparity in these findings can be hypothesized, including differences in study subject demographics (specifically ethnicity, different PCOS criteria) and clinical characteristics (i.e. higher prevalence of obesity in the present study). In our study, 
we used the 1990 NIH PCOS criteria, but the studies by Glintborg et al. (32) and Barber et al. (33) based the diagnosis of PCOS on the Rotterdam diagnostic criteria, and thus represent a more heterogenous group of women. Furthermore, inclusion criteria for the control group and the methodology used to quantify adiponectin multimers and estimate IR also differed among the studies.

Several limitations of our study should be considered. The hyperinsulinemic-euglycemic clamp technique is the most scientifically sound technique for measuring insulin sensitivity (34) and is the 'gold standard' by which other assessments are compared. Although HOMA-IR has been demonstrated to correlate well with clamp techniques and has been frequently used in clinical applications (35); this index, calculated using fasting glucose and insulin levels, may be less discriminating than other measures of insulin sensitivity. Additionally, we quantified the multimeric forms of adiponectin via an ELISA that required pretreatment with one of several proteases in order to selectively digest specific forms of adiponectin. The results obtained by this method for measuring adiponectin species have previously been shown to correlate with results obtained by quantitative western blot analysis (21). Consequently, this commercially available ELISA has also been used by other groups as well $(36,37)$. Although additional ELISA assays have been developed that measure HMW adiponectin directly $(38,39)$, results obtained from the various ELISA systems have not been directly compared.

In conclusion, the present study demonstrates that levels of both total and HMW adiponectin are decreased in Chinese women with PCOS compared with normal control women, and the differences in HMW adiponectin persist after controlling for BMI. Furthermore, HMW adiponectin is a stronger predictor of IR as estimated by HOMA-IR in both women with PCOS and normal women than total adiponectin as indicated by forward, stepwise, linear regression analysis. Although differences in IR estimated by HOMA-IR were not readily apparent between PCOS and normal groups of women in this study, levels of SHBG, a potential alternative index of IR, were significantly decreased in women with PCOS. Furthermore, total and HMW adiponectin levels demonstrated that strong inverse correlates with SHBG levels. Additionally, it is possible that alterations in adiponectin multimers may contribute to the phenotypic presentation of PCOS via metabolic pathways independent of IR. Further investigation is warranted to determine both the mechanisms by which HMW adiponectin is altered in PCOS and the potential contributions of alterations in adiponectin multimers on ovulatory dysfunction in PCOS women.

\section{Declaration of interest}

The authors declare that there is no conflict of interest that could be perceived as prejudicing the impartiality of the research reported.

\section{Funding}

This work was supported by Shanghai Renji Hospital research funds (T Tao, 2005), Shanghai Jiaotong University Outstanding teacher research funds (to W Fan, 2004), and the National Institutes of Health (K23HD053742 to E P Wickham, 2009).

\section{Acknowledgements}

We thank all the women who participated in the study. We also gratefully acknowledge the assistance of the nursing staff and physicians (in particular Dr Li) at Shanghai Renji Hospital and technical assistants who performed the biochemical analyses. Lastly, we gratefully acknowledge the valuable guidance and input of Dr John Nestler in the preparation of this manuscript.

\section{References}

1 Carmina E \& Lobo RA. Polycystic ovary syndrome (PCOS): arguably the most common endocrinopathy is associated with significant morbidity in women. Journal of Clinical Endocrinology and Metabolism $1999 \mathbf{8 4} 1897-1899$.

2 Dunaif A. Insulin resistance and the polycystic ovary syndrome: mechanism and implication for pathogenesis. Endocrine Reviews 199718 774-800.

3 Morin-Papunen LC, Vauhkonen I, Koivunen RM, Ruokonen A \& Tapanainen JS. Insulin sensitivity, insulin secretion, and metabolic and hormonal parameters in healthy women and women with polycystic ovarian syndrome. Human Reproduction 200015 1266-1274.

4 Venkatesan AM, Dunaif A \& Corbould A. Insulin resistance in polycystic ovary syndrome: progress and paradoxes. Recent Progress in Hormone Research 2001 56 295-308.

5 Barber TM, McCarthy MI, Wass JA \& Franks S. Obesity and polycystic ovary syndrome. Clinical Endocrinology $2006 \mathbf{6 5}$ 137-145.

6 Stefan N, Bunt JC, Salbe AD, Funahashi T, Matsuzawa Y \& Tataranni PA. Plasma adiponectin concentrations in children: relationships with obesity and insulinemia. Journal of Clinical Endocrinology and Metabolism 200287 4652-4656.

7 Daimon M, Oizumi T, Saitoh T, Kameda W, Hirata A, Yamaguchi H, Ohnuma H, Igarashi M, Tominaga M \& Kato T. Decreased serum levels of adiponectin are a risk factor for the progression to type 2 diabetes in the Japanese population: the Funagata study. Diabetes Care 200326 2015-2020.

8 Snehalatha C, Mukesh B, Simon M, Viswanathan V, Haffner SM \& Ramachandran A. Plasma adiponectin is an independent predictor of type 2 diabetes in Asian Indians. Diabetes Care 2003 26 3226-3229.

9 Weyer C, Funahashi T, Tanaka S, Hotta K, Matsuzawa Y, Pratley RE \& Tataranni PA. Hypoadiponectinemia in obesity and type 2 diabetes: close association with insulin resistance and hyperinsulinemia. Journal of Clinical Endocrinology and Metabolism 200186 1930-1935.

10 Hotta K, Funahashi T, Bodkin NL, Ortmeyer HK, Arita Y, Hansen BC \& Matsuzawa Y. Circulating concentrations of the adipocyte protein adiponectin are decreased in parallel with reduced insulin sensitivity during the progression to type 2 diabetes in rhesus monkeys. Diabetes 200150 1126-1133.

11 Tsao TS, Tomas E, Murrey HE, Hug C, Lee DH, Ruderman NB, Heuser JE \& Lodish HF. Role of disulfide bonds in Acrp30/adiponectin structure and signaling specificity. Different oligomers activate different signal transduction pathways. Journal of Biological Chemistry $2003 \mathbf{2 7 8} 50810-50817$.

12 Hara K, Horikoshi M, Yamauchi T, Yago H, Miyazaki O, Ebinuma H, Imai Y, Nagai R \& Kadowaki T. Measurement of the high-molecular weight form of adiponectin in plasma is useful for the prediction of insulin resistance and metabolic syndrome. Diabetes Care 200629 1357-1362. 
13 Lara-Castro C, Luo N, Wallace P, Klein RL \& Garvey WT. Adiponectin multimeric complexes and the metabolic syndrome trait cluster. Diabetes 200655 249-259.

14 Zawadski JK \& Dunaif A. Diagnostic criteria for polycystic ovary syndrome: towards a rational approach. In Current Issues in Endocrinology and Metabolism: Polycystic Ovary Syndrome, pp 377-384. Eds A Dunaif, J Givens, F Haseltine \& G Merriam, New York: Blackwell, 1992.

15 Lane DE. Polycystic ovary syndrome and its differential diagnosis. Obstetrical and Gynecological Survey 200661 125-135.

16 Adams J, Franks S, Polson DW, Mason HD, Abdulwahid N, Tucker M, Morris DV, Price J \& Jacobs HS. Multifollicular ovaries: clinical and endocrine features and response to pusatile gonadotropin releasing hormone. Lancet 19852 1375-1379.

17 World Health Organization Western Pacific Region, International Association for the Study of Obesity and International Obesity Task Force 2000. Steering Committee. Assessment diagnosis. The Asia-Pacific Perspective: Redefining Obesity and Its Treatment. 2000 pp 15-21. Health Communications Australia Pty Limited: Melbourne, Australia.

18 Reynders M, Anckaert E, Schiettecatte J \& Smitz J. Evaluation of a new automated electrochemiluminescent sex hormone-binding globulin (SHBG) immunoassay. Clinical Chemistry and Laboratory Medicine 200543 86-89.

19 Vermeulen A, Verdonck L \& Kaufman JM. A critical evaluation of simple methods for the estimation of free testosterone in serum. Journal of Clinical Endocrinology and Metabolism $1999 \mathbf{8 4}$ 3666-3372.

20 Matthews DR, Hosker JP, Rudenski AS, Naylor BA, Treacher DF \& Turner RC. Homeostasis model assessment: insulin resistance and beta-cell function from fasting plasma glucose and insulin concentrations in man. Diabetologia 198528 412-419.

21 Ebinuma H, Miyazaki O, Yago H, Hara K, Yamauchi T \& Kadowaki T. A novel ELISA system for selective measurement of human adiponectin multimers by using proteases. Clinica Chimica Acta $200637247-53$.

22 Panidis D, Kourtis A, Farmakiotis D, Mouslech T, Rousso D \& Koliakos G. Serum adiponectin levels in women with polycystic ovary syndrome. Human Reproduction 200318 1790-1796.

23 Spranger J, Möhlig M, Wegewitz U, Ristow M, Pfeiffer AF, Schill T, Schlosser HW, Brabant G \& Schofl C. Adiponectin is independently associated withinsulin sensitivity in women with polycystic ovary syndrome. Clinical Endocrinology 20046 1738-1746.

24 Haugen F \& Drevon CA. Activation of nuclear factor-kappaB by high molecular weight and globular adiponectin. Endocrinology $20071485478-5486$.

25 Schober F, Neumeier M, Weigert J, Wurm S, Wanninger J, Schaffler A, Dada A, Liebisch G, Schmitz G, Aslanidis C \& Buechler C. Low molecular weight adiponectin negatively correlates with the waist circumference and monocytic IL-6 release. Biochemical and Biophysical Research Communications 2007 361 968-973.

26 Aso Y, Yamamoto R, Wakabayashi S, Uchida T, Takayanagi K, Takebayashi K, Okuno T, Inoue T, Node K, Tobe T, Inukai T \& Nakano Y. Comparison of serum high-molecular weight (HMW) adiponectin with total adiponectin concentrations in type 2 diabetic patients with coronary artery disease using a novel enzyme-linked immunosorbent assay to detect HMW adiponectin. Diabetes 200655 1954-1960.

27 Nakashima R, Kamei N, Yamane K, Nakanishi S, Nakashima A \& Kohno N. Decreased total and high molecular weight adiponectin are independent risk factors for the development of type 2 diabetes in Japanese-Americans. Journal of Clinical Endocrinology and Metabolism 200691 3873-3877.
$28 \mathrm{Xu} \mathrm{A}$, Chan KW, Hoo RL, Wang Y, Tan KC, Zhang J, Chen B, Lam MC, Tse C, Cooper GJ \& Lam KS. Testosterone selectively reduces the high molecular weight form of adiponectin by inhibiting its secretion from adipocytes. Journal of Biological Chemistry $2005 \mathbf{2 8 0} 18073-18080$.

29 Cibula D, Skrha J, Hill M, Fanta M, Haaková L, VrbIková J \& Zivný J. Prediction of insulin sensitivity in nonobese women with polycystic ovary syndrome. Journal of Clinical Endocrinology and Metabolism $2002 \mathbf{8 7} 5821-5825$.

30 Toulis KA, Goulis DG, Farmakiotis D, Georgopoulos NA, Katsikis I, Tarlatzis BC, Papadimas I \& Panidis D. Adiponectin levels in women with polycystic ovary syndrome: a systematic review and a meta-analysis. Human Reproduction 200915 297-307.

31 Aroda V, Ciaraldi TP, Chang SA, Dahan MH, Chang RJ \& Henry RR. Circulating and cellular adiponectin in polycystic ovary syndrome: relationship to glucose tolerance and insulin action. Fertility and Sterility $2008 \mathbf{8 9} 1200-1208$.

32 Glintborg D, Frystyk J, Højlund K, Andersen KK, Henriksen JE, Hermann AP, Hagen C, Flyvbjerg A \& Andersen M. Total and high molecular weight (HMW) adiponectin levels and measures of glucose and lipid metabolism following pioglitazone treatment in a randomized placebo-controlled study in polycystic ovary syndrome. Clinical Endocrinology 200868 165-174.

33 Barber TM, Hazell M, Christodoulides C, Golding SJ, Alvey C, Burling K, Vidal-Puig A, Groome NP, Wass JA, Franks S \& McCarthy MI. Serum levels of retinol-binding protein 4 and adiponectin in women with polycystic ovary syndrome: associations with visceral fat but no evidence for fat mass-independent effects on pathogenesis in this condition. Journal of Clinical Endocrinology and Metabolism 200893 2859-2865.

34 DeFronzo RA, Tobin JD \& Andres R. Glucose clamp technique: a method for quantifying insulin secretion and resistance. American Journal of Physiology $1979237214-223$.

35 Mather KJ, Hunt AE, Steinberg HO, Paradisi G, Hook G, Katz A, Quon MJ \& Baron AD. Repeatability characteristics of simple indices of insulin resistance: implications for research applications. Journal of Clinical Endocrinology and Metabolism 200186 5457-5464.

36 Katsuki A, Suematsu M, Gabazza EC, Murashima S, Nakatani K, Togashi K, Yano Y \& Sumida Y. Decreased high-molecular weight adiponectin-to-total adiponectin ratio in sera is associated with insulin resistance in Japanese metabolically obese, normal-weight men with normal glucose tolerance. Diabetes Care 200629 2327-2328.

37 Blüher M, Brennan AM, Kelesidis T, Kratzsch J, Fasshauer M, Kralisch S, Williams CJ \& Mantzoros CS. Total and high-molecular weight adiponectin in relation to metabolic variables at baseline and in response to an exercise treatment program: comparative evaluation of three assays. Diabetes Care 200730 280-285.

38 Sinha MK, Songer T, Xiao Q, Sloan JH, Wang J, Ji S, Alborn WE, Davis RA, Swarbrick MM, Stanhope KL, Wolfe BM, Havel PJ, Schraw T, Konrad RJ, Scherer PE \& Mistry JS. Analytical validation and biological evaluation of a high molecular-weight adiponectin ELISA. Clinical Chemistry 200753 2144-2151.

39 Nakano Y, Tajima S, Yoshimi A, Akiyama H, Tsushima M, Tanioka T, Negoro T, Tomita M \& Tobe T. A novel enzyme-linked immunosorbent assay specific for high-molecular-weight adiponectin. Journal of Lipid Research 200647 1572-1582.

Received 21 May 2010

Accepted 7 June 2010 